\title{
Études comparées de la fonction poétique,
} 1981-1993

\section{Yves Bonnefoy}

\section{(2) OpenEdition \\ 1 Journals}

Édition électronique

URL : https://journals.openedition.org/annuaire-cdf/1064

DOI : 10.4000/annuaire-cdf.1064

ISBN : 978-2-7226-0325-7

ISSN : 2109-9227

Éditeur

Collège de France

Édition imprimée

Date de publication : 1 avril 2013

Pagination : 766-769

ISBN : 978-2-7226-0198-7

ISSN : 0069-5580

\section{Référence électronique}

Yves Bonnefoy, «Études comparées de la fonction poétique, 1981-1993 », L'annuaire du Collège de

France [En ligne], 112 | 2013, mis en ligne le 22 novembre 2013, consulté le 22 août 2022. URL : http:// journals.openedition.org/annuaire-cdf/1064; DOI : https://doi.org/10.4000/annuaire-cdf.1064 
Président de la Fondation des Amis des sciences (Académie des sciences).

Président du sous-groupe médical « Solidarité Japon », institué par l'Académie des sciences à la suite de la catastrophe provoquée par le tremblement de terre et le tsunami de Fukushima. Le rapport est paru aux Éditions EDP Sciences [6].

\section{Références}

[1] Baulieu E.E., « Neurosteroids : of the nervous system, by the nervous system, for the nervous system », in Conn P.M. (éd.), Recent Progress in Hormone Research, The Endocrine Society Press, Bethesda, 52, 1997, 1-32.

[2] Chambraud B., Sardin E., Giustiniani J., Dounane O., Schumacher M., Goedert M. et Baulieu E.E., "A role for FKBP52 in Tau protein function », Proc. Natl. Acad. Sci. USA, $107,2010,2658-2663$.

[3] Giustiniani J., Sineus M., Sardin E., Dounane O., Panchal M., Sazdovitch V., Duyckaerts C., Chambraud B. et Baulieu E.E., «Decrease of the immunophilin FKBP52 accumulation in human brains of Alzheimer's disease and FTDP-17 », Journal of Alzheimer Disease, 29, 2012, 471-483.

[4] Chambraud B., Sardin E., Nakatani H., Paquet D., Baulieu E.E. et Tawk M., «FKBP52 plays an essential role in driving axonal outgrowth and controls motility and spinal motor axonal morphology via inhibition of pathological Tau activity in zebrafish », publication en préparation.

[5] Bianchi M. et Baulieu E.E., «3 3 -methoxy-pregnenolone (MAP4343) as an innovative therapeutic approach for depressive disorders », Proc. Natl. Acad. Sci. USA, 109, 2012, $1713-$ 1718.

[6] Groupe de travail Solidarité Japon présidé par Alain Carpentier, avec Étienne-Émile Baulieu, L'accident majeur de Fukushima. Considérations sismiques, nucléaires et médicales. Académie des sciences, EDP Sciences, 2012.

\section{Yves BONNEFOY}

\section{Études comparées de la fonction poétique, 1981-1993}

\section{PRINCIPALES PUBlications ET ACTIVITÉS DEPUIS LE $1^{\text {er }}$ OCTOBRE 2011}

\section{Livres}

Bonnefoy Y., Sous le signe de Baudelaire, Gallimard, 2011, 414 p.

Bonnefoy Y., Je vois sans yeux et sans bouche je crie, 24 sonnets de Pétrarque, avec des dessins de Gérard Titus-Carmel, Galilée, 2011, 80 p.

Bonnefoy Y., Peintures murales de la France gothique, nouvelle édition, ELLUG, Université Stendhal, Grenoble, 2012, 159 p., avec 66 photographies de Pierre Devinoy.

Bonnefoy Y., L'inachevable, Entretiens sur la poésie, 1990-2010, nouvelle édition, Le livre de poche, avril 2012, $528 \mathrm{p}$.

Bonnefoy Y., Plusieurs raisons de peindre des arbres, Éditions de Corlevour, 2012, 80 p, avec des peintures et dessins d'Agnès Prévost. 
Bonnefoy Y., Grands rayons rouges, id. manuscrit en facsimilé, non paginé, en feuilles, Trames, 2011.

Bonnefoy Y., Bibliothèques savantes, La Grande Ourse, Plus loin, plus loin, Allo, oui ?, Encore sur l'invention du dessin, 2011, Vous encore, L'étoile sept, 2012 : livres-collages de Bertrand Dorny, réalisés par tamponnage typographique en 10 ex., signés par l'auteur et l'artiste, accompagnés d'un CD audio contenant le texte lu par l'auteur.

Bonnefoy Y. et Lafay D., Jacques Truphémus, RH Éditions, Lyon, 2011, 230 p.

Bonnefoy Y., Le Digamma, Galilée, 2012, 88 p.

\section{En revues ou dans des ouvrages collectifs}

Bonnefoy Y., «L'obstination de Chestov », dans Chestov L., Athènes et Jérusalem, Le Bruit du temps, 2011, p. 533-544.

Bonnefoy Y., «Yoshio Abé », L’Année Baudelaire, 13/14, 2009-2010, p. 13-17.

Bonnefoy Y., «L'Italien à portée de voix », Cahier italien, 2008, 2009, 2010, 2011, Società Dante Alighieri, Comitato di Parigi, s.d., p. 39-48.

Bonnefoy Y., «Miklos Bokor», dans Cahier Miklos Bokor, essais réunis par Annette Becker et Anne Bernou, Institut national d'histoire de l'art et William Blake \& Co. Edit. décembre 2011, p. 11-23.

Bonnefoy Y., « Poésie et société », dans Écrire malgré l'horreur, l'Arbre à paroles, ${ }^{\circ} 154$, 2011-2012, p. 43-45.

Bonnefoy Y., « Les œuvres de l'inconscient », Werner Lambersy, Nu(e) 50, 2012, p. 105-106.

Bonnefoy Y., «Lettre à Paula, à propos des Planches courbes »; suivi de «A Variant of Les Planches courbes », dans Paula Rego, Fondation Calouste Gubelkian, Paris, 2012, p. 36-37 et 40-41, traduit par Rudolf A.

Bonnefoy Y., «Le latin, la démocratie, la poésie », dans Suzzoni C. et Aupetit H, Sans le latin..., collection « Mille et une nuits », Fayard, avril 2012, p. 385-393.

Bonnefoy Y., « Alain Veinstein », dans « Dossier Alain Veinstein », CCP, Marseille, 2012, p. 25-32.

Bonnefoy Y., «Andrés Sànchez Robayna », dans Andrés Sànchez Robayna, Le livre, derrière la dune, Éditions du Murmure, 2012.

Bonnefoy Y., «La traduction de la poésie et l'université », dans Cheminements, Revue alsacienne de littérature, $\mathrm{n}^{\circ} 117,1^{\mathrm{er}}$ semestre 2012, p. 126-137.

Bonnefoy Y., « Dessin/couleur », dans de Asis F., Dessin/Couleur, Carnet de voyage IX, $\grave{A}$ l'atelier, 2012, p. 13-16.

Bonnefoy Y., «Préface» dans Album Amicorum, CEuvres choisies pour Arnauld Brejon de Lavergnée, Éditions Librairie des Musées, 2012, p. 3-5.

Bonnefoy Y., «Traduire. La tâche du traducteur», dans Europe, n 1000-1001, aoûtseptembre 2012 .

\section{Traductions en volume}

Bonnefoy Y., Second Simplicity : New Poetry and Prose, 1991-2011, traduit par Rogers H., Yale University Press, The Margellos World Republic of Letters, New Haven et Londres, 2012. $290 \mathrm{p}$.

Bonnefoy Y., Disorder, précédé de Poésie et Théâtre, Éditions VVV, Halifax, Canada, mars 2012, 54 p., traduit par Bishop M. 
Bonnefoy Y., The Arrière-Pays, Seagull Press, Londres, New York, Calcutta, 2012, 220 p., avec «Afterword: Septembrer 2004 », "The Place of Grasses », "My Memories of Armenia » et traduction, préface et direction de l'édition de Stephen Romer.

Bonnefoy Y., Streichend Schreiben (Raturer outre), traduit par Edl E. et Matz W., Stiftung Lyrik Kabinett, Munich, 2012.

Bonnefoy Y., Beginning and End of the Snow, traduit par Emily Grosholz, Bucknell University Press, 2012, 96 p.

Bonnefoy Y., O gibanju in negibnosti Douve (Du mouvement et de l'immobilité de Douve), traduction en slovène de Nadja Dobnik, Poetiko Nove Lire, Ljubljana, 2012, 90 p.

\section{Principales traductions en revue ou dans des ouvrages collectifs}

Bonnefoy Y., « Lectio Magistralis », dans Conferimento Laurea Honoris Causa in «Teoria e prassi della traduzione » a Yves Bonnefoy, 27 octobre 2011, Palazzo Corigliano, Naples, p. 81-99; texte italien, p. 33-51, traduit par Fusco Girard G.

Bonnefoy Y., «Eine Fotografie » et d'autres poèmes de Raturer outre dans Weltklang Nacht der Poesie, Poesie Festival Berlin 2011, p. 35-46, traduit par Edl E. et Matz W.

Bonnefoy Y., «Poemas inéditas de Yves Bonnefoy» dans Dicta \& Contradicta, Rio de Janeiro, $\mathrm{n}^{\circ} 7$, juin 2011, p. 150-157, traduit par Laranjeira M.

Bonnefoy Y., «O poeta improvàvel », entretien avec Chris Miller, id., p. 10-25, traduit par Nogueira É.

Bonnefoy Y., «La longue chaîne de l'ancre », «Le peintre dont le nom est la neige », divers sonnets », texte français et traductions de Fabio Scotto, dans Poesia XXIV, 2011, $\mathrm{n}^{\circ}$ 264, p. 6-15, précédé de Scotto F., «Yves Bonnefoy, Nel respiro del mondo ».

Bonnefoy Y., «Quelques remarques sur le travail d'Alexandre Hollan, Alcuni osservazioni sul lavoro di Alexandre Hollan» dans Alexandre Hollan, Silences en couleurs, Museo Morandi, Bologne, 2011, p. 9-20, traduit par Elefante Ch.

Bonnefoy Y., «Le grande ombre », dans Anterem, VI série, n 83, p. 84-89 (avec le texte français, « De grandes ombres », traduit par Peduzzi A.Ch.).

Bonnefoy Y., «La traducción come intercambio » et « La traducción de la poesia (1976) » dans Bolletín del Taller de Traducción literaria de la Universidad de la Laguna, automne 2011, p. 1, 3-4, traduit par Curell Cl.

Bonnefoy Y., « Die Gegenwärtige Stunde », dans Ostra-Gehege, n 65, 1, 2012, p. 20-27. traduit par Pfau U., «BriefWechsel zwischen Yves Bonnefoy und Una Pfau », id., p. 27-33.

Bonnefoy Y., «Nisida» et sa traduction en italien par Cacace V., dans Racconti per Nisida, isola d'Europa, Alfredo Guida Editore, Naples, 2012, p. 8-9.

Bonnefoy Y., «From Rue Traversière et autres récits en rêve », dans PN Review 204, vol. 38, $\mathrm{n}^{\circ}$ 4, Manchester, mars-avril 2012, p. 37-39, traduit par Beverley Bie Brahic et dans le même volume, «In conversation with Yves Bonnefoy », entretien avec Chris Miller, p. 40-45.

Bonnefoy Y., « Le photographe dans le train / Il fotografo in treno », « Une photographie / Una fotografia », « Encore une photographie / Ancora una fotografia », dans Bonnefoy Y., Trizzino L., Le photographe dans le train / Treno, Ed. Polistampa, Florence, 2012, p. 4-29, textes italiens traduits par Scotto F.

Bonnefoy Y., «My Memories of Armenia », dans Agni 75, 2012, p. 24-31, traduit par Naughton J.

Bonnefoy Y., «La seule rose », « La vie errante », « De vent et de fumée », « Les planches courbes » et d'autres poèmes, dans Vukmirovic M., Anthologie de la poésie française du $X X^{e}$ siècle, Belgrade, 2011, p. 560-579.

Bonnefoy Y. et Chris Miller, «Una conversación », dans Cuadernos Hispanoamericanos, 745-746, juillet-août 2012, p. 9-22, traduction de Clara Curell. 
Bonnefoy Y., « Varie ragioni per dipingere gli alberi », dans Lettere internazionale $\mathrm{n}^{\circ} 113$, $3^{\text {e }}$ trimestre 2012, p. 40-44, traduction en italien de Fabio Scotto.

\section{Principales autres activités}

17 octobre 2011, réception à Lausanne du Grand prix de poésie « Pierrette Micheloud ».

26 octobre, rencontre des prisonniers de Nisida à Naples

27 octobre, réception d'un doctorat honoris causa de l'Université L'Orientale à Naples et réception du Premio Napoli au Musée de Capodimonte.

17 novembre, présentation de Jacqueline Risset à l'Istituto italiano di cultura de Paris.

19 novembre, conversation avec Alain Veinstein à la Maison des écrivains.

10 janvier 2012, participation au séminaire d'Antoine Compagnon au Collège de France : «Pourquoi Baudelaire?».

25 janvier, «Poésie et archéologie », avec Jean-Paul Avice, au Colloque franco-allemand d'archéologie, à l'Université de Metz.

31 janvier, Présentation du livre Peintures murales de la France gothique à l'Université de Grenoble.

$1^{\text {er }}$ février, débat avec Daniel Lançon à l'Université de Grenoble.

18 février, «Carte blanche » à la Bibliothèque Municipale de Tours : «Écrire en l'heure présente », avec Gwenaëlle Aubry, Jean-Yves Masson et Daniel Lançon.

5 mars, réception du prix de poésie Kowalski de la Ville de Lyon à la Mairie de Lyon.

16 mars, lecture à l'École des Beaux-Arts de Tours dans la classe d'Alain Borer, avec Jacqueline Risset. - 17 mars, lecture à Chaumussay, Indre-et-Loire. - 18 mars, lecture au Château du Rivau.

2 avril, lecture et discussion avec Jérôme Thélot à la Bibliothèque de l'Arsenal.

11 mai, participation au colloque «D'autres langues que la mienne » dirigé par Michel Zink au Collège de France : «Langue, verbe, parole : les pouvoirs du parlar cantando ».

13 juin, lecture de «Raturer outre» au Lyrik Kabinett de Munich, à l'occasion de la publication de la version allemande du livre, Streichend Schreiben dans la traduction d'Elisabeth et Wolfgang Matz. Paris.

27 septembre, lecteur du «Plusieurs raisons de peindre des arbres » à la librairie Tschann,

\section{Jacques BOUVERESSE}

\section{Philosophie du langage et de la connaissance, 1995-2010}

Une part importante du travail effectué au cours de l'année 2010-2011 avait été consacrée à la préparation de l'édition électronique du texte des quatre dernières années de cours que j'ai données au Collège de France: Dans le labyrinthe: nécessité, contingence et liberté chez Leibniz (2008-2010) et Qu'est-ce qu'un système philosophique? (2006-2008). L'année 2011-2012 a été consacrée en premier lieu à l'achèvement de ce travail et le texte des cours est désormais disponible en ligne, par le site Internet du Collège de France (voir publications). La mise au point, en vue d'une édition du même type, du texte des trois années de cours que j'ai données sur 